\title{
Impact of Community Structure on Cascades
}

\author{
MEHRDAD MOHARRAMI, University of Michigan \\ VIJAY SUBRAMANIAN, University of Michigan \\ MINGYAN LIU, University of Michigan \\ MARC LELARGE, ENS/INRIA, Paris
}

\section{Extended Abstract}

In this paper we investigate a type of cascade problem on graphs that has been used to study the spread of new technology or opinions in social networks, see e.g., [Granovetter 1978]. The underlying model typically consists of a few (selected) initial adopters (nodes in the network) or "seeds" and a particular adoption model that determines the condition under which a node will choose to adopt given the states of its neighbors. A commonly studied model is the threshold model, whereby individuals adopt the new technology based on how many neighbors have already chosen it.

Prior work in this area has generally focused on analyzing what happens when the underlying network is given by a single community modeled as a sparse random graph, see e.g., [Amini 2010; Lelarge 2012]. In this paper we will instead consider graphs with a type of community structure (also known as modular networks), wherein multiple sparse random graphs are weakly interconnected. This could model, for instance, segments of the population (e.g., different age or ethnic groups), where members of a single segment are more strongly connected (with a relatively high node degree) and cross-segment connections are weak, i.e., fewer members are connected to those from a different segment. We are particularly interested in whether the existence of communities (and how different initial seeding mechanism) affects the number of individuals who eventually adopt the new technology. While earlier works have looked at this problem using heuristic methods, see e.g., [Galstyan and Cohen 2007; Gleeson 2008], we set out to present a mathematically rigorous analysis of this problem.

Specifically, we consider the permanent adoption model where nodes that have adopted the new technology cannot change their state. Our analysis presents a differential-equationbased tight approximation to the stochastic process of adoption under the threshold contagion model. The analysis of the differential equation leads to a correctness proof of a mean-field equation for the contagion in a large network, as well as an algorithm to calculate the properties of the contagion. Using this method, we are able to analyze the impact of advertising by means of seeding of the nodes with the new technology or opinion.

A full version of this paper appears at: http://arxiv.org/abs/1606.00858

V. Subramanians is partially supported by NSF via grant IIS 1538827 . He also acknowledges support by INRIA for a visit to Paris in May 2015. M. Moharrami and M. Liu are partially supported by the NSF under grants CNS 1422211 and ECCS 1446521. M. Lelarge is partially supported by ANR via grant ANR11-JS02-005-01.

Authors addresses: M. Moharrami, V. Subramanian, M. Liu, Electrical \& Computer Engineering Department, University of Michigan; email: \{moharami,vgsubram,mingyan\}@umich.edu; M. Lelarge, INRIA-Ecole Normale Superieure, email: marc.lelarge@ens.fr.

Permission to make digital or hard copies of part or all of this work for personal or classroom use is granted without fee provided that copies are not made or distributed for profit or commercial advantage, and that copies bear this notice and the full citation on the first page. Copyrights for third-party components of this work must be honored. For all other uses, contact the owner/author(s). Copyright is held by the author/owner(s).

EC'16, July 24-28, 2016, Maastricht, The Netherlands.

ACM 978-1-4503-3936-0/16/07.

http://dx.doi.org/10.1145/2940716.2953924 
We consider random graphs with given degree distribution which are locally tree-like so that the structure up to any finite depth when viewed from any node of the graph is a tree with high probability. Using mean-field approximation, the final proportion of adopters is given by $\phi=\Phi(\boldsymbol{\mu})$ where $\boldsymbol{\mu}$ is the solution to the fixed point equation $\boldsymbol{\mu}=F(\boldsymbol{\mu})$. The components of functions $\Phi(\cdot)$ and $F(\cdot)$ are defined as follows:

$$
\begin{aligned}
& F_{j, j}(\boldsymbol{\mu})=\mathbb{E}\left[D_{j}\left(1-\alpha_{D_{j}, D_{m}}^{(j)}\right) \mathbf{1}\left\{\sum_{i=1}^{D_{j}-1} Z_{i}^{(j, j)}+\sum_{i=1}^{D_{m}} Z_{i}^{(j,-j)}<K_{j}\left(D_{j}, D_{m}\right)\right\} / \mathbb{E}\left(D_{j}\right)\right] \\
& F_{-j, j}(\boldsymbol{\mu})=\mathbb{E}\left[D_{m}\left(1-\alpha_{D_{j}, D_{m}}^{(j)}\right) \mathbf{1}\left\{\sum_{i=1}^{D_{m}-1} Z_{i}^{(j,-j)}+\sum_{i=1}^{D_{j}} Z_{i}^{(j, j)}<K_{j}\left(D_{j}, D_{m}\right)\right\} / \mathbb{E}\left(D_{m}\right)\right] \\
& \Phi_{j}(\boldsymbol{\mu})=\mathbb{E}\left[\left(1-\alpha_{D_{j}, D_{m}}^{(j)}\right) \mathbf{1}\left\{\sum_{i=1}^{D_{j}} Z_{i}^{(j, j)}+\sum_{i=1}^{D_{m}} Z_{i}^{(j,-j)}<K_{j}\left(D_{j}, D_{m}\right)\right\}\right]
\end{aligned}
$$

where for every $j^{\prime}, j \in\{1,2\}, Z_{i}^{\left(j, j^{\prime}\right)}$ are i.i.d. Bernoulli random variables with parameter $1-\mu^{\left(j, j^{\prime}\right)}, D_{j}$ is a random variable with the community $j$ degree distribution, $D_{m}$ has intercommunity degree distribution, $K_{j}(\cdot, \cdot)$ is the threshold function for nodes in community $j$, and $\alpha_{d_{j}, d_{m}}^{(j)}$ is the probability of a node in community $j$ to be a seed node, given her neighbors in community $j$ and $-j$ to be $d_{j}$ and $d_{m}$ respectively.

Providing a differential-equation-based tight approximation, we prove the sample-path of contagion process can be approximated using the solution of the following differential equation:

$$
\frac{d \boldsymbol{\mu}}{d t}=F(\boldsymbol{\mu})-\boldsymbol{\mu} \quad \boldsymbol{\mu}(\mathbf{0})=\mathbf{1}, \quad \boldsymbol{\mu} \in[0,1]^{4} .
$$

Moreover, we prove that the differential equation converges to the fixed point solution of $F(\boldsymbol{\mu})=\boldsymbol{\mu}$ that is closest to $\mathbf{1}$, which is also given by $\lim _{n \rightarrow \infty} F^{n}(\mathbf{1})$. Finally, for the case of linear thresholds (as a function of the total degree) we characterize both necessary and sufficient conditions for contagion to happen. Specifically, we prove contagion happens if and only if $\rho(\nabla F(\mathbf{1}))$ is greater than 1 when $\alpha_{d_{j}, d_{m}}^{(j)}$ is set to be zero, where $\rho(\cdot)$ is the Perron-Frobenius eigenvalue of a non-negative matrix. Finally, although we discuss results for two communities, all of them can be generalized to the case of $k$ community.

\section{REFERENCES}

Hamed Amini. 2010. Bootstrap percolation and diffusion in random graphs with given vertex degrees. Electronic Journal of Combinatorics 17 (2010), R25.

Aram Galstyan and Paul Cohen. 2007. Cascading dynamics in modular networks. Physical Review E 75, 3 (2007), 036109.

James P Gleeson. 2008. Cascades on correlated and modular random networks. Physical Review E 77, 4 (2008), 046117.

Mark Granovetter. 1978. Threshold models of collective behavior. American journal of sociology (1978), 1420-1443.

Marc Lelarge. 2012. Diffusion and cascading behavior in random networks. Games and Economic Behavior 75, 2 (2012), 752-775. 